\title{
PBX3 is associated with proliferation and poor prognosis in patients with cervical cancer
}

This article was published in the following Dove Press journal:

OncoTargets and Therapy

Number of times this article has been viewed

Hongfang Li',2
Gaogao Sun'
Chang Liu'
Jing Wang
Rong Jing
Jie Wang
Xiaohuan Zhao'
Xiaoyan Xu'
Yongxiu Yang
'Department of Obstetrics and
Gynecology, The First People's
Hospital of Lanzhou City,
'Department of Gynecology, The
First Hospital of Lanzhou University,
Lanzhou, 'Department of Gynecology,
Longhua District People's Hospital of
Shenzhen City, Shenzhen, ${ }^{4}$ Department
of Obstetrics and Gynecology,
The First Affiliated Hospital of Sun
Yat-sen University, Guangzhou,
'Department of Gynecology, Tianjin
Fifth Central Hospital, Tianjin,
People's Republic of China

Abstract: Pre-B-cell leukemia homeobox 3 (PBX3) is upregulated in various malignancies; however, the role of PBX3 in cervical cancer (CC) is unknown. The purpose of this study was to explore the expression characteristics, clinicopathological significance, and molecular biological function of PBX3 in CC. The expression levels of PBX3 were analyzed in CC cell lines and tumor specimens by real-time polymerase chain reaction (RT-PCR), Western blotting, and immunohistochemical staining. The clinicopathological characteristics associated with PBX3 expression were evaluated. An RNA interference approach was employed to suppress PBX3 expression in $\mathrm{CC}$ in vitro and in vivo, determine its role in cell proliferation and analyze its molecular function. We found that PBX3 expression was significantly upregulated in CC cell lines and clinical specimens compared with normal cells and adjacent nontumorous cervical tissues. PBX3 was an independent predictive factor of poor prognosis, and its expression was correlated with tumor diameter, pathological grading, lymph node metastasis, invasion depth, vascular invasion, and clinical stage of CC. Multivariate analysis suggested that PBX3 expression may represent an independent prognostic indicator of the survival of $\mathrm{CC}$ patients. CC patients with high PBX3 expression exhibited reduced overall survival compared with those with low PBX3 expression. Additionally, stable downregulation of PBX3 expression in CC cell lines suppressed cell proliferation and decreased p-AKT protein expression levels in vitro. Similarly, in vivo assays demonstrated that $\mathrm{PBX} 3$ downregulation in $\mathrm{CC}$ cells markedly inhibited tumor size and weight. Overall, we demonstrated that $\mathrm{PBX} 3$ can promote $\mathrm{CC}$ cell proliferation via the AKT signaling pathway and that it may serve as a prognostic marker. Our data indicate that inactivation of PBX3 may be an effective clinical treatment for CC.

Keywords: cervical cancer, pre-B-cell leukemia homeobox 3, RNA interference, proliferation, AKT signaling pathway

\section{Introduction}

Cervical cancer (CC) is the second most frequent malignant disease in women, which causes cancer-associated mortality worldwide, ${ }^{1,2}$ with $\sim 529,000$ new cases and 275,000 deaths every year. ${ }^{3}$ Despite improvements in diagnostic and therapeutic strategies, the 5-year survival rate for patients with advanced stage $\mathrm{CC}$ remains poor, because of recurrence and metastasis after surgery and concurrent chemoradiotherapy. ${ }^{4,5}$ Hence, comprehensive understanding of the molecular mechanisms underlying CC progression and identification of therapeutic targets are crucial. ${ }^{6-8}$

The newly discovered protein, pre-B-cell leukemia homeobox 3 (PBX3), a member of the recently described PBX family, is consistently reported as associated with tumor growth and progression. ${ }^{9}$ Increased expression of PBX3 is closely correlated with tumor growth and progression in malignancies including malignant prostate cancer, ${ }^{10}$ leukemia, ${ }^{9,11}$ and gastric cancer, ${ }^{12,13}$ suggesting that PBX3 is associated with

Correspondence: Yongxiu Yang Department of Obstetrics and Gynecology, The First People's Hospital of Lanzhou City, Lanzhou 730000,

People's Republic of China

Tel/fax +86 93। 2337286

Email yongxiuyang@163.com 
several types of cancer. ${ }^{14}$ However, the biological function of PBX3 in CC has not been investigated..$^{15}$ The purpose of this study was to investigate the potential of PBX3 to act as a biomarker in $\mathrm{CC}$ and to provide a better understanding of the molecular pathophysiology of $\mathrm{CC}$, with the aim of facilitating the development of improved methods for its detection, diagnosis, and gene therapy.

\section{Materials and methods}

\section{Samples and patients}

The current retrospective study enrolled 190 patients diagnosed with primary $\mathrm{CC}$ who underwent surgical resection in The First People's Hospital of Lanzhou City and The First Affiliated Hospital of Lanzhou University from June 2006 to July 2012. The mean patient age was $47.26 \pm 9.41$ years (range 23-71 years). The date of death and the date of relapse were used to calculate estimates of overall survival (OS). Eight paired samples of CC tissues and their corresponding adjacent nontumorous cervical tissues (ANTs) were collected, immediately frozen in liquid nitrogen, and stored at $-80^{\circ} \mathrm{C}$ until use. All paraffin-embedded and fresh tissues were obtained for research purposes with the consent of each patient. Written informed consent was obtained from all patients, and research protocols were approved by the Clinical Research Ethics Committees of The First People's Hospital of Lanzhou City and The First Affiliated Hospital of Lanzhou University. The Clinical Research Ethics Committees approved the protocol. Clinical information is summarized in Table 1.

\section{Cell lines, siRNA vectors, and retroviral infection}

Human cervical cells (normal cervical cells [NCECs]) and CC cell lines, including CaSki, Hela, C-33A, Siha, HT-3, and ME- 180 , were cultured at $37^{\circ} \mathrm{C}$ in $5 \% \mathrm{CO}_{2}$ in RPMI1640 medium containing 10\% fetal bovine serum (FBS). These cell lines were purchased from the Shanghai Institutes for Biological Sciences, Chinese Academy of Sciences (Shanghai, China). To inhibit intracellular expression of PBX3 protein, two ShRNA oligonucleotides (RNAi\#1 5'-GAAAGAGGGAGCAAAGGTTCT-3'; RNAi\#2 5'-GTGTCACAGAGTCTTGTAAAG-3') were designed and cloned into the pCMV3 vector to generate pCMV3PBX3-ShRNA1 and pCMV3-PBX3-ShRNA2, respectively. Retroviral production and infection were performed as described previously, ${ }^{16}$ were infected for 48 hours, enabling them to stably express PBX3-shRNA. Then, cells were selected with $0.5 \mu \mathrm{g} / \mathrm{mL}$ Neomycin for 10 days.
Table I Expression of PBX3 in samples from patients with cervical cancer and its relationship with clinical pathological parameters ( $\mathrm{n}[\%$ of total])

\begin{tabular}{|c|c|c|c|c|}
\hline & $\begin{array}{l}\text { High } \\
\text { expression }\end{array}$ & $\begin{array}{l}\text { Low } \\
\text { expression }\end{array}$ & $\chi^{2}$ value & $P$-value \\
\hline$n=190$ & $103(54.21)$ & 87 (45.79) & & \\
\hline Age & & & 47.66 & 0.08 \\
\hline$\leq 45$ years & $46(55.42)$ & $37(44.58)$ & & \\
\hline$>45$ years & $57(53.27)$ & $50(46.73)$ & & \\
\hline HPV infection & & & 23.7 & 0.07 \\
\hline Negative & $18(5 \mid .43)$ & $17(48.57)$ & & \\
\hline Positive & $85(54.84)$ & $70(45.16)$ & & \\
\hline Pathological grading & & & 32.6 & 0.00 \\
\hline$I+I I$ & $49(39.84)$ & $74(60.16)$ & & \\
\hline III & $54(80.6)$ & $13(19.4)$ & & \\
\hline Tumor in diameter & & & 21.9 & 0.01 \\
\hline$>4 \mathrm{~cm}$ & $63(64.29)$ & 35 (35.7I) & & \\
\hline$\leq 4 \mathrm{~cm}$ & $40(43.48)$ & $52(56.52)$ & & \\
\hline Lymph node metastasi & & & 22.79 & 0.00 \\
\hline No & $43(46.74)$ & $49(53.26)$ & & \\
\hline Yes & $60(6 \mid .22)$ & 38 (38.78) & & \\
\hline Invasion depth & & & 37.87 & 0.00 \\
\hline Shallow interstitial & $37(48.68)$ & 39 (5I.32) & & \\
\hline Deep interstitial & $66(57.89)$ & $48(42.11)$ & & \\
\hline Involved with vessel & & & 29.67 & 0.02 \\
\hline No & 51 (46.79) & $58(53.21)$ & & \\
\hline Yes & $52(64.2)$ & $29(35.8)$ & & \\
\hline Clinical stage & & & 22.66 & 0.03 \\
\hline FIGO early & $53(5 \mathrm{I} .46)$ & $50(48.54)$ & & \\
\hline FIGO advanced & $50(57.47)$ & $37(42.53)$ & & \\
\hline
\end{tabular}

Abbreviations: FIGO, International Federation of Gynecology and Obstetrics; HPV, human papillomavirus; PBX3, pre-B-cell leukemia homeobox 3.

\section{Real-time polymerase chain reaction (RT-PCR)}

Total RNA samples were extracted from cultured cells and fresh tissues using TRIzol reagent (Thermo Fisher Scientific, Waltham, MA, USA) according to the manufacturer's instructions. RNA samples were pretreated with RNase-free DNase. Reverse transcription of $2 \mu \mathrm{g}$ of total RNA from each sample was performed using a QuantiTect Reverse Transcription Kit (Qiagen NV, Venlo, the Netherlands) following the manufacturer's protocol. SYBR Green reagent (Thermo Fisher Scientific) was used for RT-PCR to analyze mRNA expression ${ }^{17}$ and to quantify the amount of $P B X 3$ mRNA in CC cell lines relative to the NCECs and in each primary CC tissue sample, relative to their paired ANT samples. Reactions were performed in triplicate, and expression data were normalized to the geometric mean of the expression level of the housekeeping gene, GAPDH. Primer Express v 2.0 software (Thermo Fisher Scientific) was used for primer design. PCR primer sequences were designed according to the reference literature ${ }^{18}$ as follows: $P B X 3$ forward, 
5'-CTCCCAAATTCTGGGGACATG-3' and reverse, 5'-ATCCACCTGTGACTGCACATTG-3'; ${ }^{\prime 1}$ and GAPDH forward, 5'-AGGCTGTTGGCAAAGTGATTC-3' and reverse, 5'-CTTTTCCCAAACGCACAGTCA-3'.

\section{Western blotting}

Proteins were extracted from cultured cells at $70 \%-80 \%$ confluence, or from tumor/ANT tissues, separated by SDSPAGE, and transferred to polyvinylidene fluoride (PVDF) membranes. After incubation with primary monoclonal rabbit antibodies at $4^{\circ} \mathrm{C}$ for $12 \mathrm{~h}$ at room temperature, membranes were washed three times with tris-buffered saline and Tween 20 TBST) solution and then incubated with horseradish peroxidase-labeled secondary antibody (anti-rabbit IgG, 1:4,000, Sigma-Aldrich Co., St Louis, MO, USA) for $3 \mathrm{~h}$. Membranes were washed with TBST and detected proteins visualized using ECL prime Western blotting detection reagent (Amersham Biosciences, Uppsala, Sweden). ${ }^{19,20}$ Rabbit anti-PBX3 antibody (1:1,000, ab56239) was from Abcam (Cambridge, MA, US), rabbit anti-pAKT (Ser473) (1:500, SAB4504331) was from Sigma, rabbit anti-total AKT (1:1,000, SAB4500797) was from Sigma, and antiGAPDH (1:1,500, ab70136) was from Abcam.

\section{Immunohistochemistry (IHC) analysis}

IHC analysis was used to study altered protein expression in eight tumor/ANT tissue samples and 190 human CC tissues. Briefly, $4 \mu \mathrm{m}$-thick paraffin sections of patient $\mathrm{CC}$ tissue samples were baked at $65^{\circ} \mathrm{C}$ for $30 \mathrm{~min}$ and then incubated with rabbit anti-PBX3 antibody (1:1,000, ab56239). After washing, sections were incubated with horseradish peroxidase-labeled secondary antibody (anti-rabbit IgG, 1:4,000, Sigma), followed by nuclei stain. Images were acquired by Microscope (FV1000; Olympus Corporation, Tokyo, Japan). Three independent pathologists analyzed the results of immunohistochemical staining for PBX3 protein and were blinded to sample origins. The degree of immunostaining of formalin-fixed, paraffin-embedded sections was independently evaluated. The scores given by the three independent pathologists were averaged and were based on both the proportion of positively stained cells and the intensity of staining. The proportion of cells was scored as follows: $1,<10 \%$ positive tumor cells; $2,10 \%-50 \%$ positive tumor cells; $3,50 \%-75 \%$ positive tumor cells; and $4,>75 \%$ positive tumor cells. The intensity of staining was graded according to the following criteria: 0 , no staining; 1 , weak staining = light yellow; 2 , moderate staining = yellow brown; and 3, strong staining $=$ brown. The staining index was calculated as the product of the proportion of positive cells and the staining intensity score (range, $0-12$ ). ${ }^{16}$ Cutoff values for PBX3 were chosen on the basis of a measure of heterogeneity using the log-rank test with respect to OS. The optimal cutoff value was determined as follows: a staining index score of $\geq 4.3$ was used to define tumors with high PBX3 expression, while $<4.3$ indicated low PBX3 expression.

\section{Cell proliferation assay}

Hela and Siha cell lines were seeded at a density of $1 \times 10^{3}$ cells per well in 96-well plates. Cells were treated after incubation for $24 \mathrm{~h}$ at $37^{\circ} \mathrm{C}$ in a $5 \% \mathrm{CO}_{2}$ incubator in RPMI- 1640 complete medium. The MTT assay was performed after $1,2,3,4$, and 5 days of cell culture. Culture medium was discarded, cells washed three times with phosphate-buffered saline (PBS), and $25 \mu \mathrm{L} 0.5 \%$ (w/v) MTT added into each well. The $96-$ well plates were incubated for $3 \mathrm{~h}$ at $37^{\circ} \mathrm{C}$, and then the culture medium discarded. The 96-well plates were washed three times with PBS, $120 \mu \mathrm{L}$ DMSO added into each well, incubated with slow shaking for $0.5 \mathrm{~h}$, and the absorbance at $490 \mathrm{~nm}$ was measured using an enzyme-linked immunosorbent assay reader (Multiskan FC; Thermo Fisher Scientific). ${ }^{21}$ Each experiment was repeated three times.

\section{Anchorage-independent growth ability assay}

The anchorage-independent growth ability of transfectants was analyzed by soft agar colony assay. Five hundred cells were prepared in $2 \mathrm{~mL}$ of $0.3 \%$ agar in RPMI- 1640 complete medium and layered onto $2 \mathrm{~mL} \mathrm{1 \%}$ agar in RPMI-1640 medium containing $10 \%$ FBS. The colonies were incubated for 10 days. $^{22}$ The experiment was performed three times independently for each cell line.

\section{Colony formation assay}

Colony formation assays were performed by seeding cells at concentrations of $5 \times 10^{2} /$ well in six-well plates and incubating for 10 days. Colonies were stained with $1 \%$ crystal violet. ${ }^{23}$ The experiment was performed three times independently for each cell line.

\section{Xenografted tumor model}

NOD/SCID mice (male, 6 weeks old, 20-22 g) were obtained from Vital River Laboratories (Beijing, China). All animal experiments were executed according to the National Institutes of Health Guide for the Care and Use of Laboratory Animals. Experimental procedures were approved by the Institutional Animal Care and Use Committee of The First 
People's Hospital of Lanzhou City (IACUC). Animals were treated humanely for all experimental procedures. All animals were lawfully acquired and their retention and use were in compliance with federal, state, and local laws and regulations, and in accordance with the guidelines of the IACUC guide for care and use of laboratory animals. Immunodeficient mice were randomly divided into six groups ( $n=6$ per group). Stably transfected cells $\left(1 \times 10^{7}\right)$ were injected subcutaneously into the dorsal right flank of NOD/SCID mice. The cells included Hela and Siha CC cells stably expressing ShRNA-vector, PBX3-RNAi\#1, and PBX3-RNAi\#2, respectively. Tumors were examined every 5 days, length $(L)$ and width $(W)$ were measured, and tumor volumes calculated using the equation $\left(L \times W^{2}\right) / 2$. On day 30, animals were killed, and tumors harvested, weighed, and used for protein quantification. ${ }^{24}$

\section{Statistical analysis}

All experiments were repeated three times. Data are expressed as mean \pm standard deviation (SD). Data analysis was carried out using SPSS 13.0 software (SPSS, Inc., Chicago, IL, USA). $P$-values were calculated by one-way analysis of variance followed by Tukey's post hoc test. $P<0.05$ was considered to indicate a statistically significant difference.

\section{Results}

\section{Expression level of PBX3 is significantly increased in CC cells and tissues}

The expression levels of both $\mathrm{PBX} 3$ protein and mRNA were markedly upregulated in multiple $\mathrm{CC}$ cell lines, including CaSki, Hela, C-33A, Siha, HT-3, and ME-180, compared with those in the normal cervical cell line, NCEC (Figure 1A and B). Immunohistochemical analysis confirmed that PBX3 protein levels were markedly upregulated in all eight $\mathrm{CC}$ samples compared with the matched normal ANT samples (Figure 2A). Moreover, Western blot assays indicated that $\mathrm{PBX} 3$ protein was also overexpressed in $\mathrm{CC}$ tissues compared with the paired normal ANT tissues (Figure 2B). $P B X 3$ upregulation in these clinical samples was further confirmed by RT-PCR analysis (Figure 2C). As shown in Figure 2A, there was clear staining for PBX3 in CC tissues. In contrast, no, or weak, visible staining was observed in the matched ANT tissue samples. Overall, these results clearly demonstrate that PBX3 is highly expressed in CC cells and tissues.

\section{Association of PBX3 expression and CC clinical characteristics}

To explore the potential application of determination of PBX3 levels for early diagnosis and prognosis in $\mathrm{CC}$, we analyzed the correlation between PBX3 expression
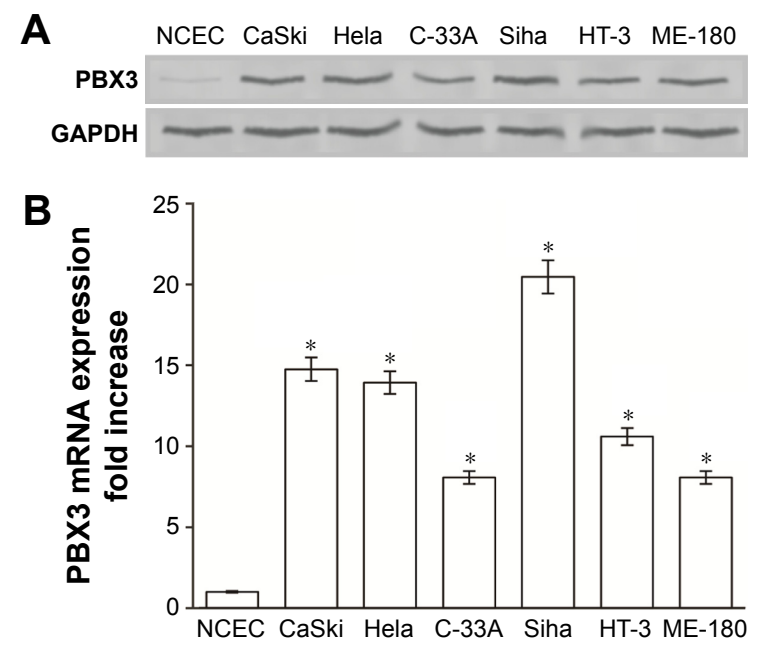

Figure I Overexpression of PBX3 mRNA and protein in cervical cancer cell lines. Notes: Western blotting $(\mathbf{A})$ and RT-PCR $(\mathbf{B})$ were used to examine the expression of PBX3 protein and mRNA in CC cell lines (CaSki, Hela, C-33A, Siha, HT-3, and ME-180) and human cervical cells (NCECs). Expression levels were normalized against those of GAPDH. Error bars represent the SD of the mean (SD) calculated from three parallel experiments. $* P<0.05$.

Abbreviations: RT-PCR, real-time polymerase chain reaction; NCECs, normal cervical cells; PBX3, pre-B-cell leukemia homeobox 3; SD, standard deviation.

and patient clinicopathological features and survival. Immunohistochemical analysis demonstrated that PBX3 was significantly overexpressed in the cytoplasm of $\mathrm{CC}$ cells. Statistical analysis revealed that PBX3 was strongly expressed in 54.21\% (103/190) of patients with CC and was markedly associated with tumor diameter, pathological grade, lymph node metastasis, invasion depth, vascular invasion, and clinical stage (all $P<0.05$ ) (Table 1). The relationship between the clinicopathological parameters of $\mathrm{CC}$ and $\mathrm{PBX} 3$ expression is summarized in Table 1.

There was no apparent relationship between PBX3 expression and other clinicopathological parameters, including human papillomavirus (HPV) infection and age (all $P>0.05$ ).

\section{PBX3 expression is significantly associated with prognosis in patients with CC}

Kaplan-Meier analysis and the log-rank test were used to determine the relationship between PBX3 expression and survival in patients with CC. The log-rank test showed that the survival time was significantly different between patients stratified into groups with low and high PBX3 expression. Moreover, Kaplan-Meier survival curves and log-rank tests demonstrated that the OS of patients with high levels of PBX3 was significantly poorer than of patients with low levels ( $P<0.05$; Figure 2D). The cumulative 5-year survival rate was $73.9 \%(95 \% \mathrm{CI}, 69.8 \%-78.4 \%)$ in the low PBX3 group, whereas it was only $40.4 \%$ (95\% CI, 38.3\%-47.1\%) in the high PBX3 group. 
A

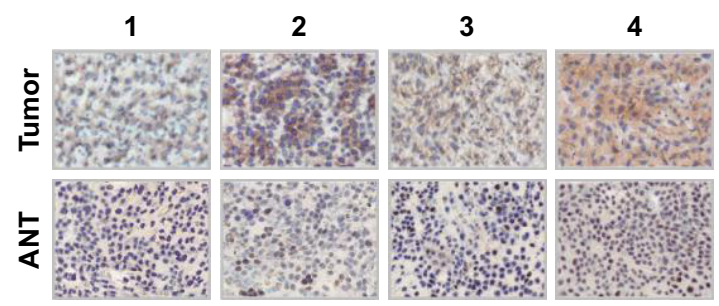

5

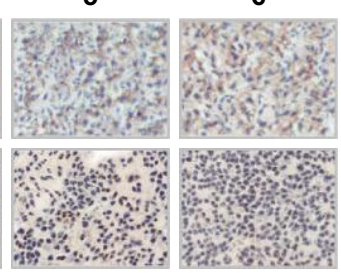

7

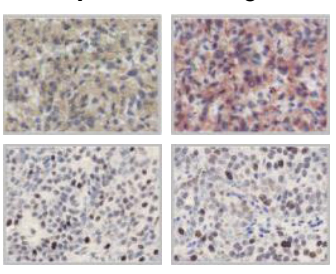

B

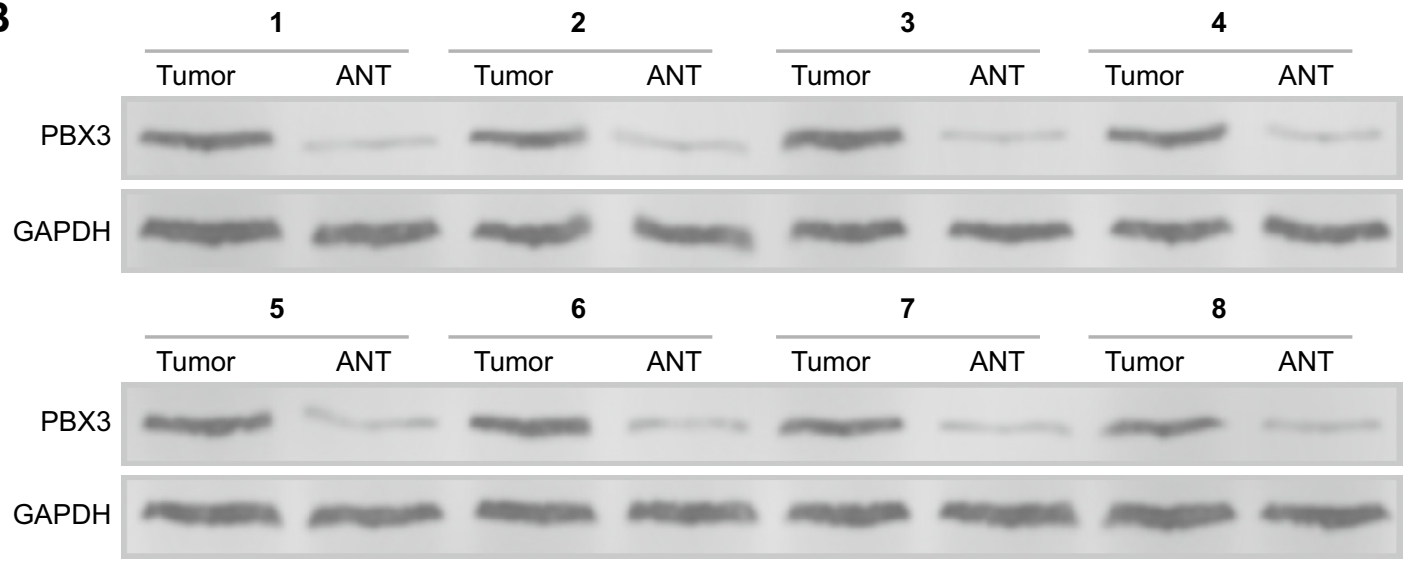

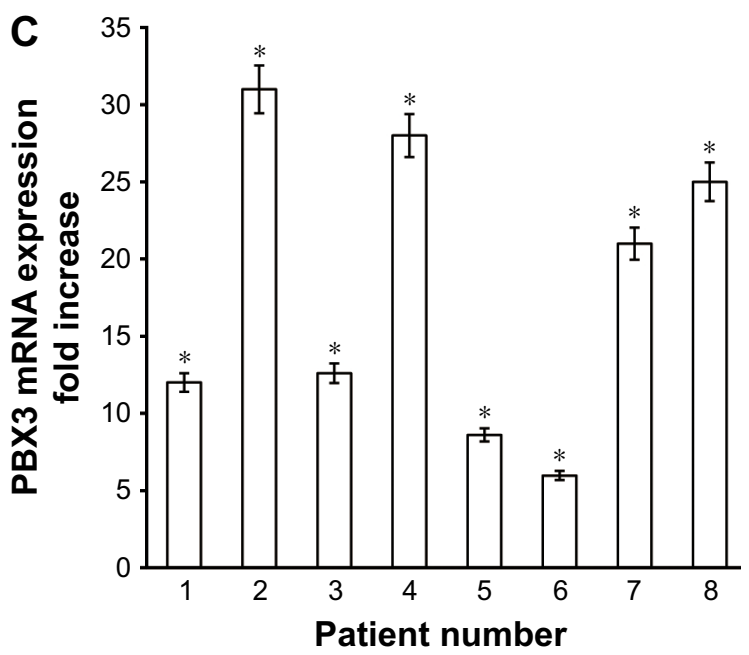
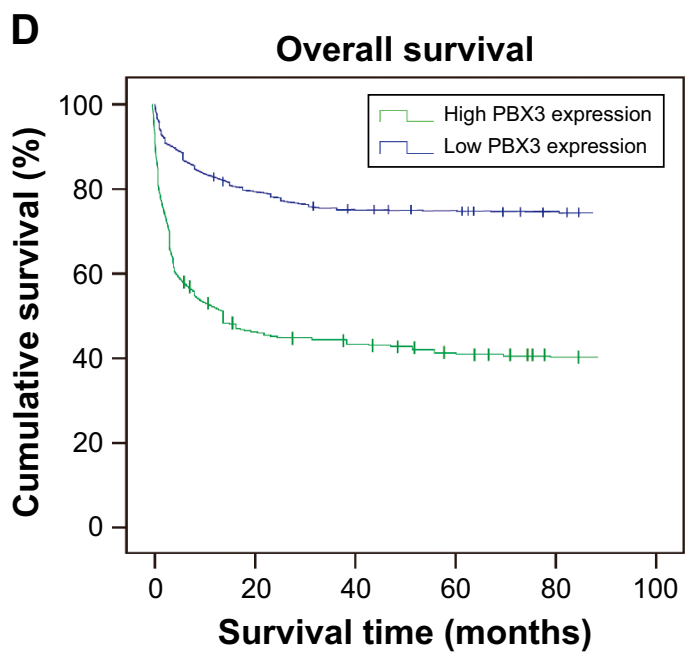

Figure 2 Overexpression of pre-B-cell leukemia homeobox 3 (PBX3) mRNA and protein in primary cervical cancer tissues.

Notes: (A) Immunohistochemical assay of PBX3 protein expression in eight pairs of matched cervical cancer (CC) cancer (tumor) and adjacent nontumor cervical tissues (ANT). (B) Western blotting assay of PBX3 protein expression in eight pairs of CC (tumor) and adjacent (ANT) tissues. (C) Average T/N ratios of PBX3 mRNA expression in paired cervical cancer $(\mathrm{T})$ and adjacent noncancerous tissues $(\mathrm{N})$ were quantified by real-time polymerase chain reaction (RT-PCR) and normalized against GAPDH. Error bars represent the standard deviation (SD) of the mean (SD) calculated from three parallel experiments. (D) Kaplan-Meier curves generated by univariate analyses (log-rank) of patients with CC with low PBX3 expression versus those with high PBX3 expression. The cumulative 5 -year survival rate was $73.9 \%$ in the low PBX3 protein expression group $(n=87)$, while it was only $40.4 \%$ in the high-expression group $(n=103) ; * P<0.05$.

Further univariate and multivariate analyses were employed to investigate correlations between PBX3 expression and other clinicopathological parameters. A Cox regression model revealed that $\mathrm{PBX} 3$ expression, tumor diameter, pathological grading, lymph node metastasis, and International Federation of Gynecology and Obstetrics (FIGO) clinical stage were independent prognostic factors associated with survival (Table 2); therefore, PBX3 may be a useful marker for prediction of OS of patients with CC.
These data show that PBX3 expression is correlated with the prognosis of patients with $\mathrm{CC}$ and a potentially effective diagnostic marker for this malignancy.

\section{PBX3 promotes CC proliferation in vitro}

As high expression of PBX3 in Hela and Siha cells has been confirmed, we infected Hela and Siha cells with two vectors expressing shRNAs that stably inhibit endogenous PBX3 expression or empty vector as a control, and then evaluated 
Table 2 Prognostic factors for patients with cervical cancer determined by Cox-regression analysis

\begin{tabular}{|c|c|c|c|c|c|c|}
\hline \multirow[t]{2}{*}{ Variable } & \multicolumn{3}{|c|}{ Univariate analysis } & \multicolumn{3}{|c|}{ Multivariate analysis } \\
\hline & $P$-value & Risk ratio & $95 \% \mathrm{Cl}$ & $P$-value & Risk ratio & $95 \% \mathrm{Cl}$ \\
\hline PBX3 expression; low vs high & 0.013 & 3.100 & $1.353-6.193$ & 0.014 & 3.215 & $1.350-6.268$ \\
\hline Tumor diameter; $>4 \mathrm{~cm}$ vs $\leq 4 \mathrm{~cm}$ & 0.015 & 4.221 & $1.214-7.592$ & 0.020 & 2.264 & $1.577-5.880$ \\
\hline Pathological grading; I + II vs III & 0.024 & 3.728 & $1.886-6.252$ & 0.018 & 3.652 & $2.097-6.151$ \\
\hline Lymph node metastasis; no vs yes & 0.011 & 2.386 & $1.078-5.110$ & 0.012 & 2.740 & $\mathrm{I} .68 \mathrm{I}-5.837$ \\
\hline FIGO clinical stage; early vs advanced & 0.025 & 2.907 & $1.146-5.084$ & 0.032 & 3.121 & $1.265-6.324$ \\
\hline
\end{tabular}

Abbreviations: $\mathrm{Cl}$, confidence interval; FIGO, International Federation of Gynecology and Obstetrics; PBX3, pre-B-cell leukemia homeobox 3.

the effect of inhibition of PBX3 on CC cell proliferation. As the plasmid vector contained a puromycin resistance sequence, we were able to generate stable CC cell lines and determine the expression levels of PBX3 mRNA and protein. As expected, RNAi\#1 and RNAi\#2 downregulated the level of PBX3 protein in the PBX3-silenced Hela and Siha cells (Figure 3A). To study the effect of PBX3 protein on cell proliferation, we determined the proliferation rates of Hela and Siha cells transfected with the shRNA-vector control and those in which PBX3 was silenced, using an MTT assay (Figure 3B). Silencing of PBX3 markedly decreased the proliferation rate of cells infected with PBX3-siRNA, compared with controls transduced with empty shRNA-vector, on day $5(P<0.05)$ (Figure 3B). Anchorage-independent growth assays confirmed the results of the MTT assays, demonstrating that reduction of endogenous PBX3 protein in Hela and Siha cells led to a markedly clear inhibition of cell growth, as indicated by a reduction in both colony number and size on soft agar in PBX3-silenced cells (Figure 3C). Furthermore, colony formation assays verified these results; colony forming ability was dramatically inhibited by the downregulation of PBX3 protein expression levels in PBX3-silenced Hela and Siha cells compared with corresponding vector only controls (Figure 3D).

To clarify the molecular mechanism underlying the effect of PBX3 CC cell proliferation, we determined the expression levels of phosphorylated AKT (p-AKT) by Western blot analysis and found that the levels of p-AKT protein were significantly decreased in the PBX3-silenced Hela and Siha cells compared with controls (Figure 3A).

\section{PBX3 enhances the tumorigenicity of $C C$ in vivo}

To confirm the in vitro experimental results, the biological role of PBX3 in CC progression was investigated using an in vivo subcutaneous tumor model to detect whether PBX3 expression could enhance the tumorigenicity of $\mathrm{CC}$ cells in vivo. As shown in Figure 4A, the subcutaneous tumor nodules formed by Hela/PBX3-ShRNA and Siha/PBX3ShRNA cells grew at significantly slower rates than those formed by shRNA-vector infected Hela and Siha control cells. Additionally, PBX3-silenced Hela and Siha cell tumors were clearly smaller than the controls (Figure 4B and C).

To determine whether the inhibition of $\mathrm{CC}$ nodule proliferation is due to the reduction of $\mathrm{PBX} 3$ protein expression, we used Western blotting experiments to detect the expression level of $\mathrm{PBX} 3$ protein in tumor tissues and found that $\mathrm{PBX} 3$ levels were significantly decreased in the PBX3-silenced Hela and Siha tumors (Figure 4D).

In summary, the results of the in vivo experiments were consistent with the in vitro data and reveal that $\mathrm{PBX} 3$ has an important role in promoting the tumorigenicity of $\mathrm{CC}$ cells in vitro and in vivo.

\section{Discussion}

$\mathrm{CC}$ is one of the most common malignant tumors in women. ${ }^{25}$ As a result of continuous progress in development of diagnosis methods, surgical therapy, radiotherapy, and chemotherapy, the incidence of $\mathrm{CC}$ has decreased worldwide. ${ }^{26}$ Nevertheless, there are still nearly 500,000 new cases and $\sim 300,000$ deaths from CC globally every year. ${ }^{27}$ Particularly in developing countries, such as China, $\mathrm{CC}$ ranks as the second most commonly diagnosed cancer and the third leading cause of cancer death for women. ${ }^{28}$ Therefore, the development of new molecular methods to improve the diagnosis of $\mathrm{CC}$ and facilitate gene therapy is urgently required..$^{29}$ No sensitive biomarkers specific for the early detection and prognosis of CC have been reported to date. ${ }^{30}$ Therefore, it is important to identify new molecular markers of CC to facilitate more accurate prediction of clinical outcomes and prescription of effective treatment. ${ }^{31}$

In the present study, multivariate analysis revealed that PBX3 protein expression level is a potential independent prognostic factor and early diagnosis marker for CC (Tables 1 and 2). To investigate the relationship between $\mathrm{PBX} 3$ and $\mathrm{CC}$, we analyzed PBX3 mRNA and protein 

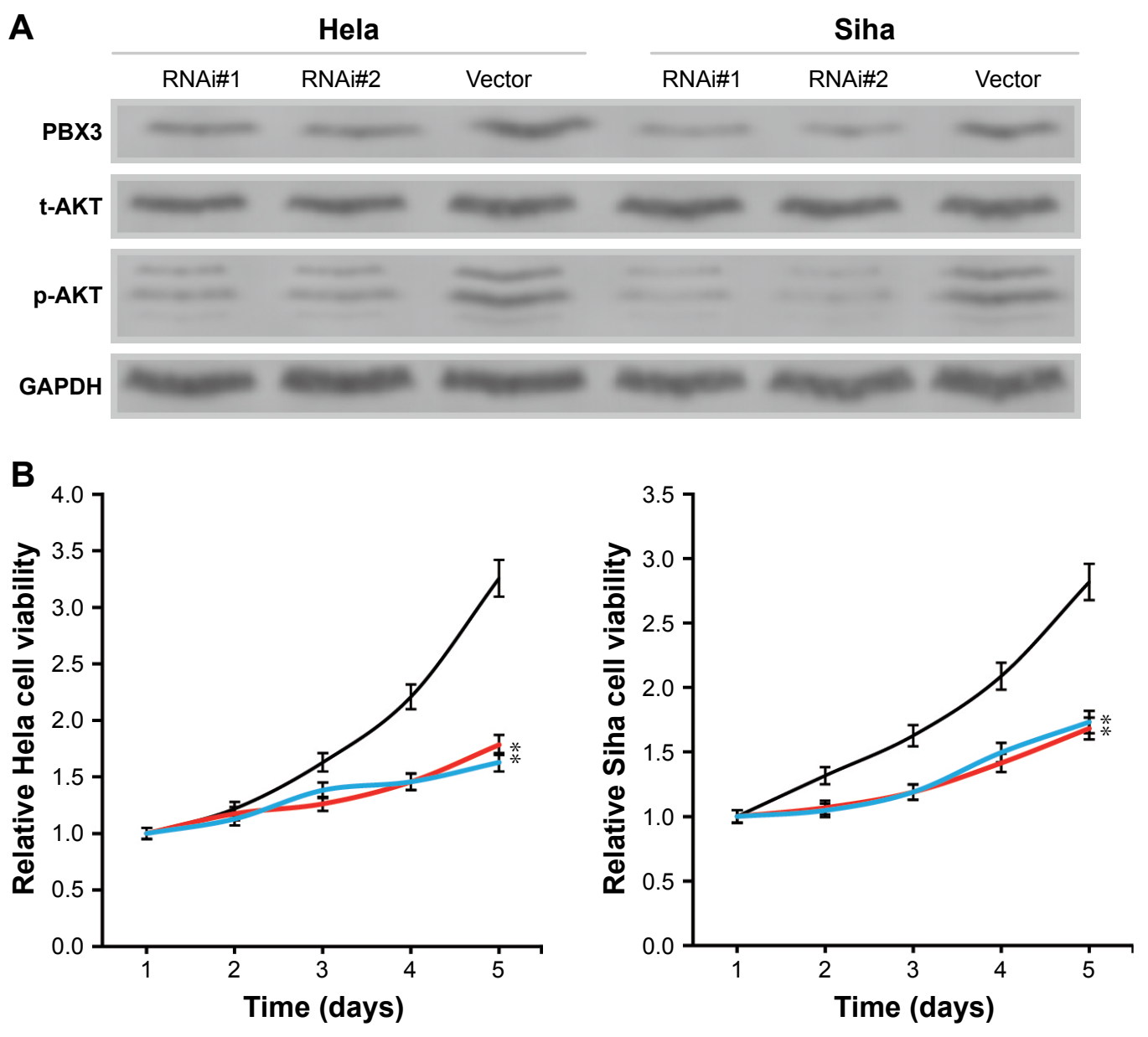

- Vector - RNAi\#1 - RNAi\#2

C

Hela

Siha
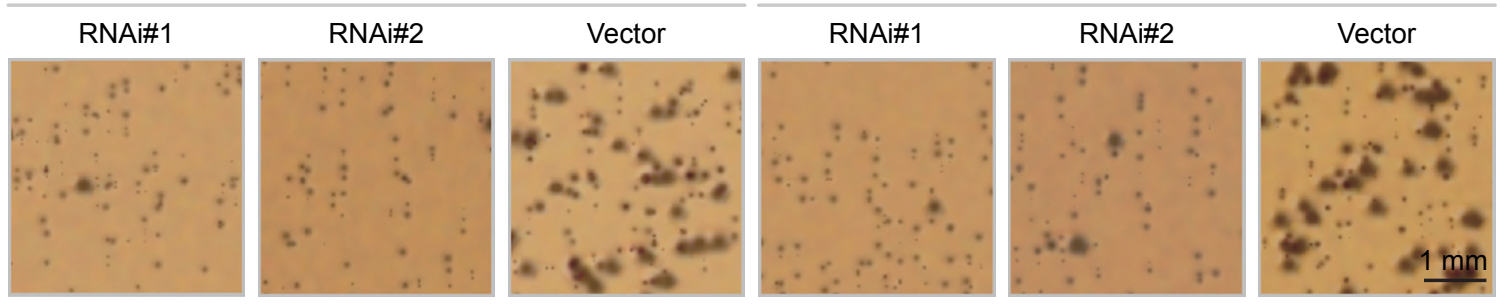

D

Hela
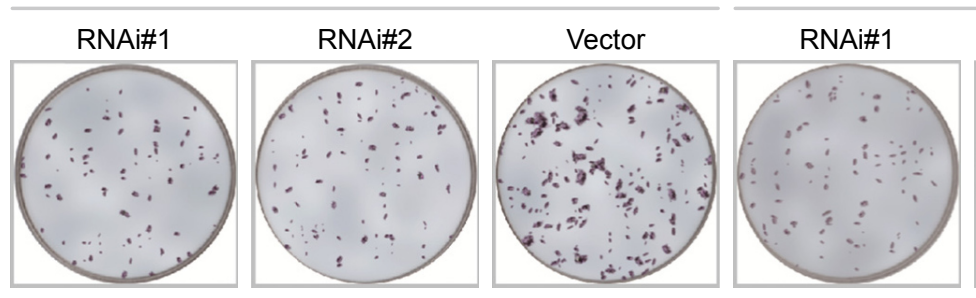

Siha

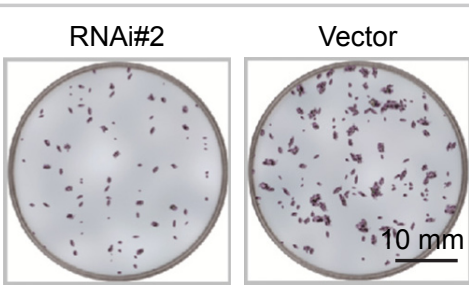

Figure 3 Pre-B-cell leukemia homeobox 3 (PBX3) plays a key role in cervical cancer (CC) cell proliferation and tumorigenicity in vitro.

Notes: (A) Western blotting analysis of PBX3, total AKT (t-AKT), and phosphorylated AKT (p-AKT), in Hela and Siha cells transfected with empty ShRNAvector and with PBX3silenced. GAPDH was used as a loading control. (B) Results of MTT assay demonstrating that growth rates of PBX3-silenced cells were decreased compared with controls treated with empty ShRNA vector. Absorbance values at days I-5 were normalized to that at day 0 (control, I00\%). Each bar represents the mean \pm SD of three independent experiments. $* P<0.05$. (C) Anchorage-independent growth assays of cells with PBX3silenced and controls treated with ShRNA vector. The number of colonies with a diameter larger than $0.1 \mathrm{~mm}$ was quantified after 10 days of culture. (D) Colony formation assays demonstrating that growth rates were decreased in PBX3-silenced cells. The number of colonies was quantified in the colony formation assay. 
A

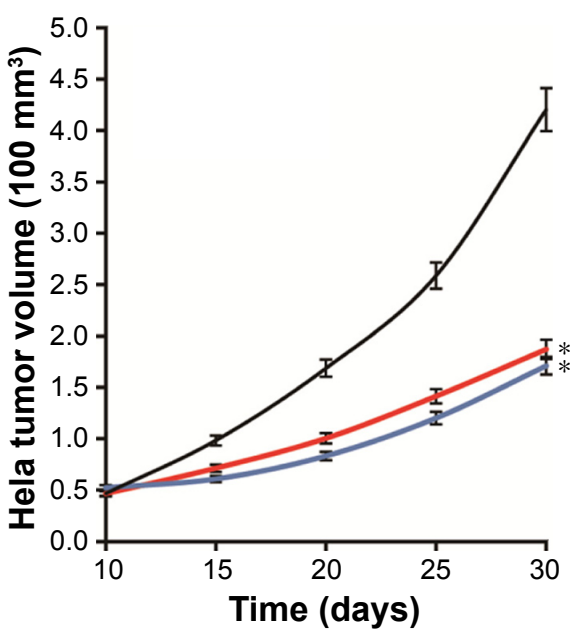

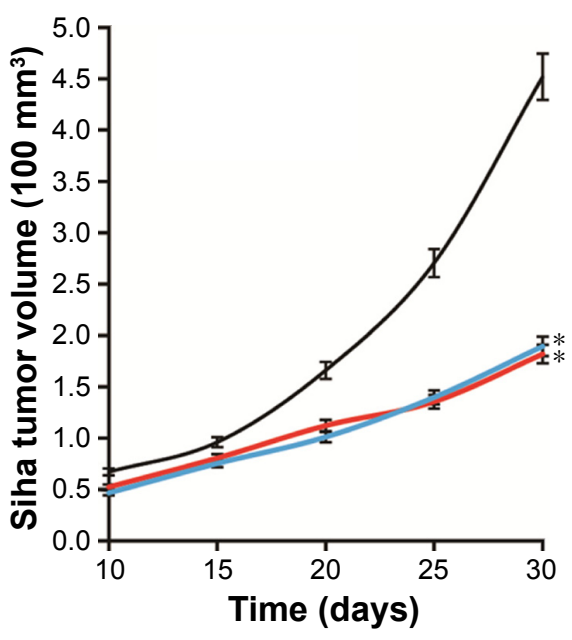

Time (days)

B
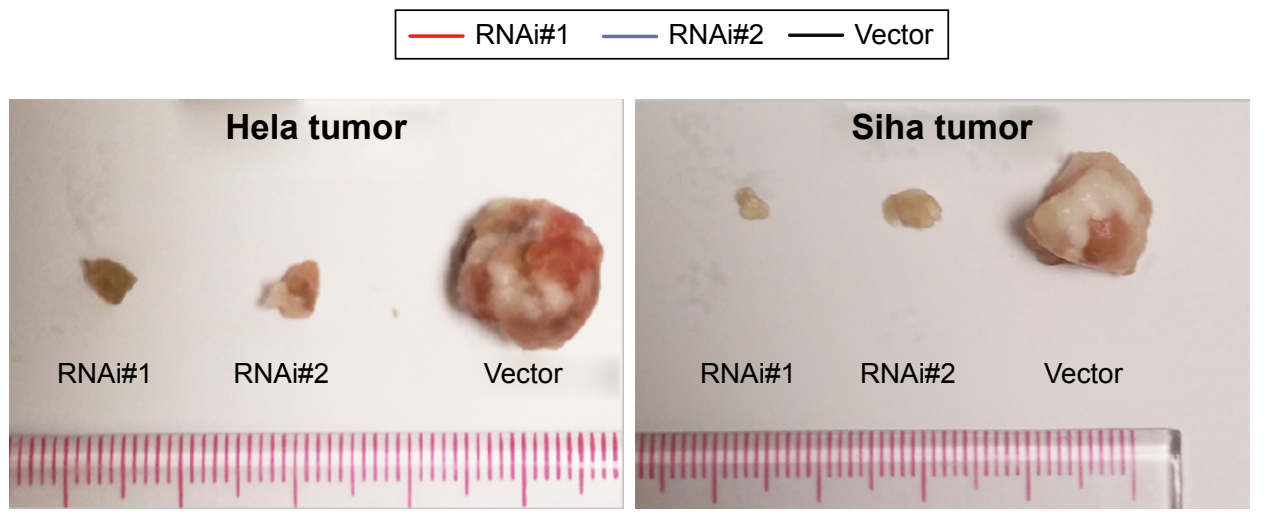

C
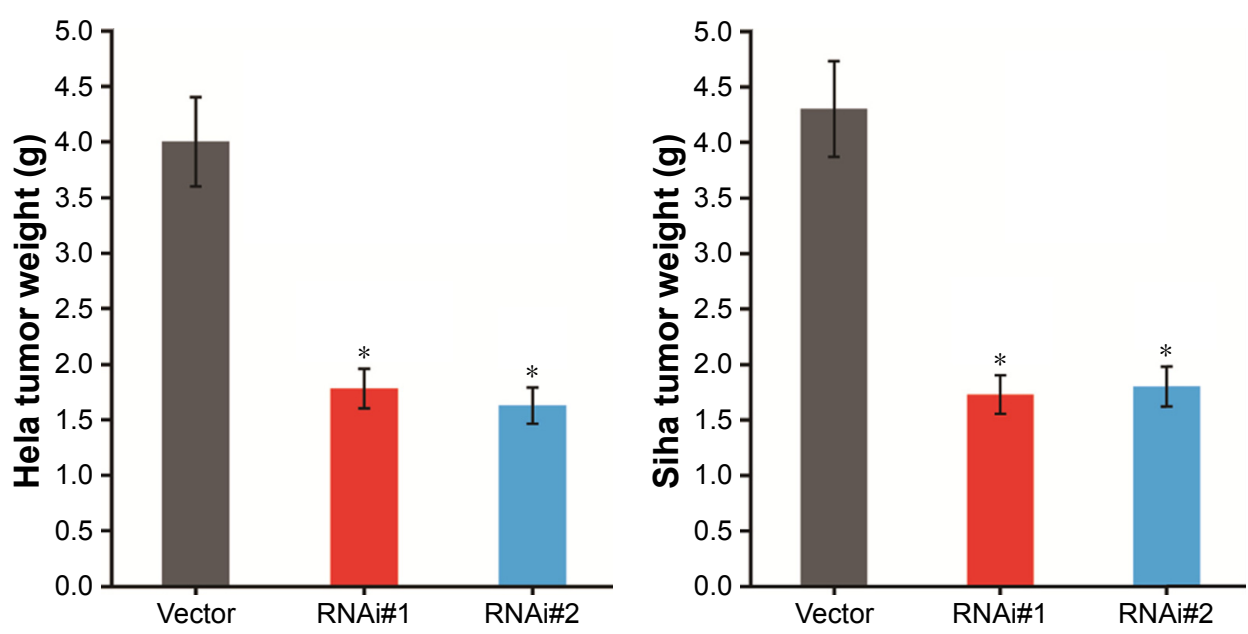

D

Hela tumor

Siha tumor

\begin{tabular}{|c|c|c|c|c|c|c|}
\hline & RNAi\#1 & RNAi\#2 & Vector & RNAi\#1 & RNAi\#2 & Vector \\
\hline PBX3 & $=$ & 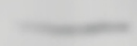 & 0 & $-2=0$ & $=$ & 6 \\
\hline GAPDH & $\mathrm{sen}$ & 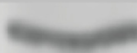 & $\cos ^{2}$ & 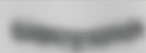 & s. & $\mathrm{cos}=$ \\
\hline
\end{tabular}

Figure 4 Downregulation of pre-B-cell leukemia homeobox 3 (PBX3) represses cervical cancer (CC) proliferation and tumorigenicity in vivo: the effect of PBX3 on tumor formation in a nude mouse xenograft model.

Notes: (A) Growth curve of subcutaneous tumors generated from PBX3 shRNA\#I and \#2-transduced Hela and Siha cells in nude mice compared with those generated from ShRNA vector-transduced Hela and Siha cells $(n=6)$. Tumor volumes were measured on the indicated days. (B) Representative photographs illustrating tumor growth in nude mice subcutaneously inoculated with Hela and Siha cells transduced with ShRNA vector, PBX3 shRNA\#I, or PBX3 shRNA\#2. (C) Mean tumor weights. Each bar represents the mean \pm SD of three independent experiments. $* P<0.05$. (D) The expression level of PBX3 protein in tumor tissues was detected by $W$ estern blot assay. 
expression levels in six CC cell lines, CC tissues from 190 cases, and eight matched fresh clinical $\mathrm{CC}$ and healthy tissue samples. We first determined that PBX3 was upregulated in six CC cell lines (Figure 1). Immunohistochemical, Western blot, and RT-PCR assays confirmed that high levels of PBX3 were expressed in eight fresh CC tissues compared with the levels in paired normal tissues at both the mRNA and protein levels (Figure 2A-C). Moreover, based on immunohistochemical analysis, we proved that high PBX3 protein expression was correlated with poor prognosis in CC (Figure 2D and Table 1). Additionally, immunostaining demonstrated that the expression level of PBX3 protein in histological sections was significantly associated with tumor diameter, pathological grading, lymph node metastasis, invasion depth, vascular invasion, and clinical stage $(P<0.05)$ (Table 1).

These results prompted us to study the molecular mechanisms underlying the role of PBX3 in CC. We hypothesized that reduction of $\mathrm{PBX} 3$ may inhibit the proliferation of $\mathrm{CC}$ and demonstrated that downregulation of PBX3 in CC cells significantly suppressed tumor proliferation in vitro and in vivo (Figure 3). Further in vivo experiments demonstrated that the growth of subcutaneous tumors was inhibited by reduction of PBX3 expression (Figure 4). The results of this study are consistent with those of previous studies of PBX3 in other types of tumor cells. ${ }^{32-35} \mathrm{PBX} 3$ has been postulated as a tumor antigen associated with immune response and immunoregulation in breast cancer ${ }^{36}$ and embryonic development. ${ }^{37}$ Therefore, we intend to explore the potential of PBX3 as a potential target for immunotherapy in future investigations.

To clarify the role of PBX3 in CC, we studied its involvement in molecular signaling pathways. AKT signaling pathways are associated with proliferation of numerous malignancies, including CC. ${ }^{38-40}$ Moreover, the role of PBX3 in gastric cancer is closely related to AKT signaling. ${ }^{13}$ Accordingly, we explored the effects of PBX3 downregulation on the levels of AKT phosphorylation in Hela and Siha cells. Downregulation of PBX3 expression reduced the levels of AKT phosphorylation in Hela and Siha cells. Synthesis of our results and those of the foregoing studies lead us to conclude that PBX3 can promote proliferation of CC cells via the AKT signaling pathway.

\section{Conclusion}

The present study illustrates that PBX3 acts as an oncogene by promoting proliferation via AKT signaling in $\mathrm{CC}$ cells from various sources and is a potential new diagnostic and prognostic marker in CC. PBX3 also has potential for development as a target for gene therapy of CC.

\section{Acknowledgments}

This study was supported by the Key research and development plan of Gansu Province (No 17yf1 fa141), Grants of the National Natural Science Foundation of China (No 81602723), the Science and Technology Project of Guangdong Province (No 2016A020215214, 2017A020215125), and the Guangdong Medical Research Foundation (A2015130). The funders had no role in study design, data collection and analysis, decision to publish, or preparation of the manuscript.

\section{Disclosure}

The authors report no conflicts of interest in this work.

\section{References}

1. Aranda S, Berkley S, Cowal S, et al. Ending cervical cancer: a call to action. Int J Gynaecol Obstet. 2017;138(suppl 1):4-6.

2. Chen L, Pan S, Ou R, et al. Distribution of pelvic lymph nodes as well as preoperative and surgical pathologic factors associated with nodal metastases in women with cervical cancer. Int J Clin Exp Pathol. 2017; 10(4):4801-4806.

3. Sawaya GF, Huchko MJ. Cervical cancer screening. Med Clin North Am. 2017;101(4):743-753.

4. Jouglar E, Thomas L, de la Rochefordiere A, et al. Toxicity and early clinical outcomes in cervical cancer following extended field helical tomotherapy to para-aortic lymph nodes. Cancer Radiother. 2016; 20(8):794-800.

5. Carneiro BA, Elvin JA, Kamath SD, et al. FGFR3-TACC3: a novel gene fusion in cervical cancer. Gynecol Oncol Rep. 2015;13:53-56.

6. Ouyang F, Liu J, Xia M, et al. GINS2 is a novel prognostic biomarker and promotes tumor progression in early-stage cervical cancer. Oncol Rep. 2017;37(5):2652-2662.

7. Peng L, Yuan X, Jiang B, Tang Z, Li GC. LncRNAs: key players and novel insights into cervical cancer. Tumour Biol. 2016;37(3):2779-2788.

8. Tang L, Yu H, Zhong W, Yu F. PTP4A3 expression is associated with the clinical features and prognosis of cervical cancer. Int J Clin Exp Pathol. 2017;10(3):3272-3278.

9. Guo H, Chu Y, Wang L, et al. PBX3 is essential for leukemia stem cell maintenance in MLL-rearranged leukemia. Int J Cancer. 2017; 141(2):324-335.

10. Ramberg H, Grytli HH, Nygard S, et al. PBX3 is a putative biomarker of aggressive prostate cancer. Int J Cancer. 2016;139(8):1810-1820.

11. Garcia-Cuellar MP, Steger J, Fuller E, Hetzner K, Slany RK. Pbx3 and Meis1 cooperate through multiple mechanisms to support Hox-induced murine leukemia. Haematologica. 2015;100(7):905-913.

12. Li B, Zhang S, Shen H, Li C. MicroRNA-144-3p suppresses gastric cancer progression by inhibiting epithelial-to-mesenchymal transition through targeting PBX3. Biochem Biophys Res Commun. 2017;484(2):241-247.

13. Wang $\mathrm{S}, \mathrm{Li} \mathrm{C}$, Wang $\mathrm{W}$, Xing C. PBX3 promotes gastric cancer invasion and metastasis by inducing epithelial-mesenchymal transition. Oncol Lett. 2016;12(5):3485-3491.

14. Han H, Du Y, Zhao W, et al. PBX3 is targeted by multiple miRNAs and is essential for liver tumour-initiating cells. Nat Commun. 2015; 6:8271.

15. LiZ, Chen P, Su R, et al. PBX3 and MEIS1 cooperate in hematopoietic cells to drive acute myeloid leukemias characterized by a core transcriptome of the MLL-rearranged disease. Cancer Res. 2016;76(3):619-629.

16. Wang J, Ou J, Guo Y, et al. TBLR1 is a novel prognostic marker and promotes epithelial-mesenchymal transition in cervical cancer. $\mathrm{Br} J$ Cancer. 2014;111(1):112-124.

17. Guo Y, Chen W, Wang W, et al. Simultaneous diagnosis and gene therapy of immuno-rejection in rat allogeneic heart transplantation model using a T-cell-targeted theranostic nanosystem. ACS Nano. 2012; 6(12):10646-10657. 
18. Chang YW, Chen JY, Lu MX, et al. Selection and validation of reference genes for quantitative real-time PCR analysis under different experimental conditions in the leafminer Liriomyza trifolii (Diptera: Agromyzidae). PLoS One. 2017;12(7):e181862.

19. Jing Z, Heng W, Xia L, et al. Downregulation of phosphoglycerate dehydrogenase inhibits proliferation and enhances cisplatin sensitivity in cervical adenocarcinoma cells by regulating Bcl-2 and caspase-3. Cancer Biol Ther. 2015;16(4):541-548.

20. Guo Y, Wang J, Li H, et al. Mediator subunit 23 overexpression as a novel target for suppressing proliferation and tumorigenesis in hepatocellular carcinoma. J Gastroenterol Hepatol. 2015;30(6):1094-1103.

21. Song R, Cong L, Ni G, et al. MicroRNA-195 inhibits the behavior of cervical cancer tumors by directly targeting HDGF. Oncol Lett. 2017; 14(1):767-775.

22. Yadav SS, Prasad SB, Prasad CB, et al. CXCL12 is a key regulator in tumor microenvironment of cervical cancer: an in vitro study. Clin Exp Metastasis. 2016;33(5):431-439.

23. Mao L, Zhang Y, Mo W, Yu Y, Lu H. BANF1 is downregulated by IRF1-regulated microRNA-203 in cervical cancer. PLoS One. 2015; 10(2):e117035.

24. Guo Y, Wang J, Zhang L, et al. Theranostical nanosystem-mediated identification of an oncogene and highly effective therapy in hepatocellular carcinoma. Hepatology. 2016;63(4):1240-1255.

25. Barrington DA, Dilley SE, Landers EE, et al. Distance from a comprehensive cancer center: a proxy for poor cervical cancer outcomes? Gynecol Oncol. 2016;143(3):617-621.

26. Mitiku I, Tefera F. Knowledge about cervical cancer and associated factors among 15-49 year old women in Dessie Town, Northeast Ethiopia. PLoS One. 2016;11(9):e163136.

27. Stecklein SR, Jhingran A, Burzawa J, et al. Patterns of recurrence and survival in neuroendocrine cervical cancer. Gynecol Oncol. 2016; 143(3):552-557.

28. Luvero D, Plotti F, Aloisi A, et al. Patients treated with neoadjuvant chemotherapy + radical surgery + adjuvant chemotherapy in locally advanced cervical cancer: long-term outcomes, survival and prognostic factors in a single-center 10-year follow-up. Med Oncol. 2016; 33(10): 110 .

29. Wang W, Chu HJ, Liang YC, et al. FABP5 correlates with poor prognosis and promotes tumor cell growth and metastasis in cervical cancer. Tumour Biol. 2016;37(11):14873-14883.
30. Jiamset I, Hanprasertpong J. Risk factors for parametrial involvement in early-stage cervical cancer and identification of patients suitable for less radical surgery. Oncol Res Treat. 2016;39(7-8):432-438.

31. Mahmoodi P, Motamedi H, Seyfi ASM, Bahrami SM, Kargar M. Molecular detection and typing of human Papilloma viruses in paraffinembedded cervical cancer and pre-cancer tissue specimens. Iran J Cancer Prev. 2016;9(1):e3752.

32. Li Z, Zhang Z, Li Y, et al. PBX3 is an important cofactor of HOXA9 in leukemogenesis. Blood. 2013;121(8):1422-1431.

33. Milech N, Kees UR, Watt PM. Novel alternative PBX3 isoforms in leukemia cells with distinct interaction specificities. Genes Chromosomes Cancer. 2001;32(3):275-280.

34. Qin P, Haberbusch JM, Soprano KJ, Soprano DR. Retinoic acid regulates the expression of $\mathrm{PBX} 1, \mathrm{PBX} 2$, and $\mathrm{PBX} 3$ in $\mathrm{P} 19$ cells both transcriptionally and post-translationally. J Cell Biochem. 2004;92(1): 147-163.

35. Ramberg H, Alshbib A, Berge V, Svindland A, Tasken KA. Regulation of PBX3 expression by androgen and Let-7d in prostate cancer. $\mathrm{Mol}$ Cancer. 2011;10:50.

36. Zheng T, Wang A, Hu D, Wang Y. Molecular mechanisms of breast cancer metastasis by gene expression profile analysis. Mol Med Rep. 2017;16(4):4671-4677.

37. Selleri L, DiMartino J, van Deursen J, et al. The TALE homeodomain protein $\mathrm{Pbx} 2$ is not essential for development and long-term survival. Mol Cell Biol. 2004;24(12):5324-5331.

38. Bahrami A, Hasanzadeh M, Hassanian SM, et al. The potential value of the PI3K/Akt/mTOR signaling pathway for assessing prognosis in cervical cancer and as a target for therapy. J Cell Biochem. 2017;5(5): 1602-1609.

39. Lu D, Qian J, Li W, et al. Beta-hydroxyisovaleryl-shikonin induces human cervical cancer cell apoptosis via PI3K/AKT/mTOR signaling. Oncol Lett. 2015;10(6):3434-3442.

40. Xu H, Zhu J, Hu C, Song H, Li Y. Inhibition of microRNA-181a may suppress proliferation and invasion and promote apoptosis of cervical cancer cells through the PTEN/Akt/FOXO1 pathway. J Physiol Biochem. 2016;72(4):721-732.
OncoTargets and Therapy

\section{Publish your work in this journal}

OncoTargets and Therapy is an international, peer-reviewed, open access journal focusing on the pathological basis of all cancers, potential targets for therapy and treatment protocols employed to improve the management of cancer patients. The journal also focuses on the impact of management programs and new therapeutic agents and protocols on

\section{Dovepress}

patient perspectives such as quality of life, adherence and satisfaction The manuscript management system is completely online and includes a very quick and fair peer-review system, which is all easy to use. Visit http://www.dovepress.com/testimonials.php to read real quotes from published authors. 